\title{
INVESTIGATING THE POTENTIAL CONSEQUENCES OF THE MEMBERSHIP FUNCTIONS IN A FUZZY LOGIC CONTROLLER-BASED OBSTACLE CLIMBING ROBOT
}

\author{
K. Alhaf Malik ${ }^{1}$, Dr.D.Elayaraja ${ }^{2}$, Dr. S. Jafar Ali Ibrahim ${ }^{3 *}$, Dr. N.S. Kalyan Chakravarthy ${ }^{4}$ \\ ${ }^{1}$ King Saud University, KSA \\ ${ }^{2,3,4}$ QIS College of Engineering and Technology, Ongole, Andra Pradesh, India \\ Email: ${ }^{3}$ jafartheni@gmail.com
}

\begin{abstract}
Autonomous mobile robots are the robots which can perform desired tasks in unstructured environment without continuous human guidance. This paper deals with the design of an Autonomous obstacle climbing robot that will able to climb an obstacle. This obstacle climbing navigation system is utilized by the intelligent fuzzy logic controller. The controller has 2 inputs and single output system. The inputs are slope and terrain type and the output is the speed of the robot. The membership functions are the building blocks of fuzzy logic system and play a vital role in the performance of mobile robot. This paper describes the effect of different membership functions namely triangular, trapezoidal membership functions are considered.
\end{abstract}

Keywords: Fuzzy logic control, Obstacle climbing robot, membership function

\section{Introduction}

These days, various mobile robots are being developed and researched in a number of fields. Mobility is an essential element of the mobile robots. The travel system for the mobile robots can be classified in two types, leg and wheel. The leg locomotion is more suitable for rough terrain. It has disadvantages of low-speed relative to wheels, complexity of control and manufacture, inefficient weight.

On the contrary to the leg locomotion, wheel driving systems can travel at high speed. Also, they are easy to control and manufacture and with light weight, but low adaptability to a rough terrain.

Researchers used fuzzy logic control for the control of mobile robots [1],[5],[8],[13],[14]. It is used in controlling of leader follower robot [2], unicycle mobile robot [4], Kephera robot [3], line follower robot [7] and automated guided vehicles [11]. Single fuzzy logic controller is used for most fuzzy logic controller. Dual fuzzy logic controller [8] and Four fuzzy logic controller combined are used [12].

\section{Materials and Methods}

The robot consists of Rocker, Bogie, Horizontal solid member, Geared motor, Microcontroller, Motor drive, remote controller.

The robot is allowed to move over a different terrain such smooth, rough and very rough etc with different slope angles ranges from -45 degrees to 45 degrees. In this research, different shape of the membership functions such as triangle, trapezoidal, Gbell shape and Gauss shape with five linguistic variables and considered and the fuzzy control system is continued with all these membership function shapes with three linguistic variables each. The results are compared and found out the output value from fuzzy logic controller which is very close the experimental value.

\section{Obstacle climbing robot}

The mechanism used in obstacle climbing Robot is Rocker bogie mechanism. This mechanism permits the robot to climb obstacles and travel in an uneven terrain. It also helps to keep its six wheels in contact with the uneven surface. These robots are utilized in the situation where human cannot go and safe.

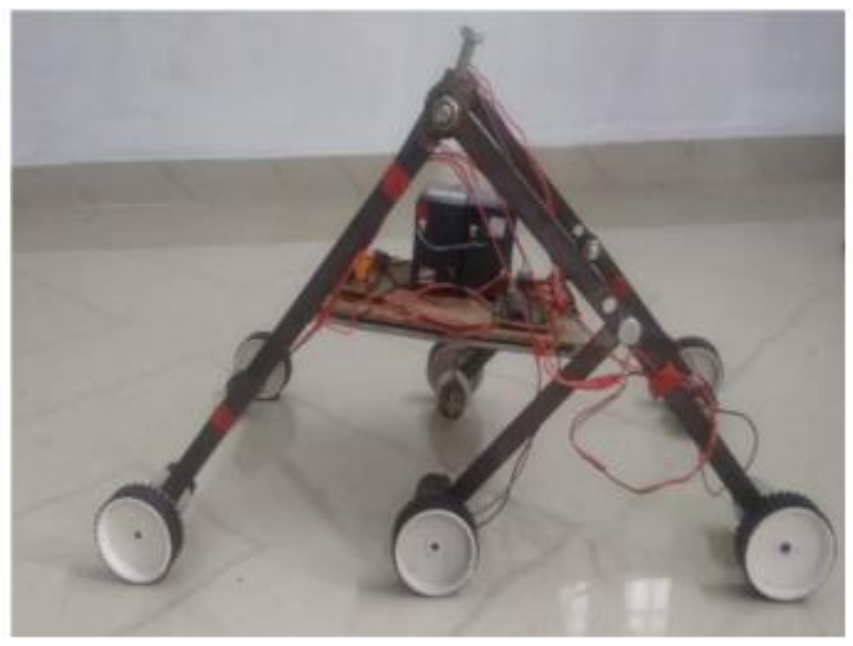

Fig.1 Obstacle climbing robot

4. Fuzzy logic control of obstacle climbing robot

The fuzzy logic control system is used to make the obstacle climbing robot an autonomous one. The input 
membership functions are slope and terrain type. The output membership function is speed. The controller used is Mamdani fuzzy controller.

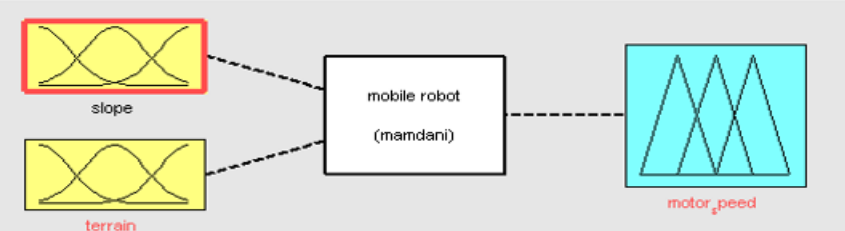

Fig.2 Fuzzy interface system of the mobile robot

\subsection{Triangular shape membership function with five} linguistic variables

The shape of the membership considered is Triangular membership function. It has five linguistic variables.Fig. 3 shows the triangular shape input membership function slope with five linguistic variables namely large negative, negative, level, positive and large positive.Fig.4 shows output membership function motor speed with five linguistic variables namely very slow, slow, medium, positive and large positive. Fig.5 shows rule view of Triangular shape membership function with five linguistic variables. The rule view clearly shows that the surface roughness value is 50 microns, motor speed is 11 miles per hour for the triangular shape membership function with five linguistic variables.

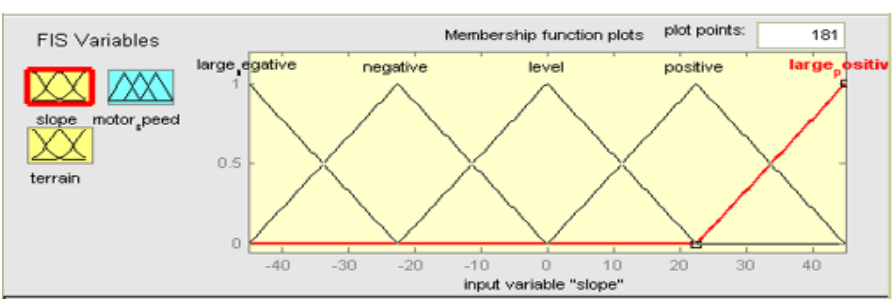

Fig.3 Triangular shape input membership function slope with five linguistic variables

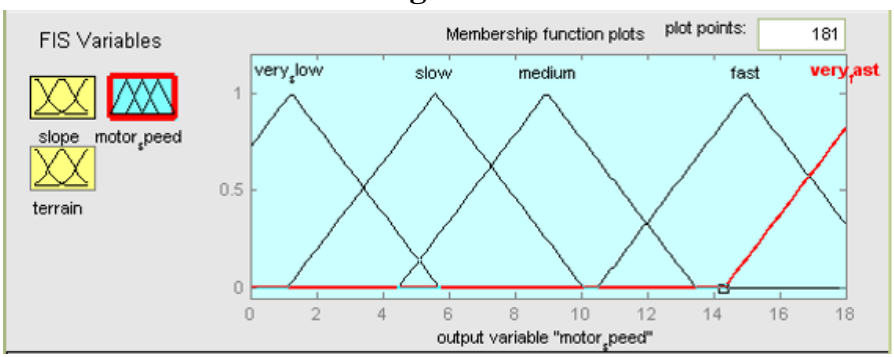

Fig.4 Triangular shape output Motor speed with five membership function variables

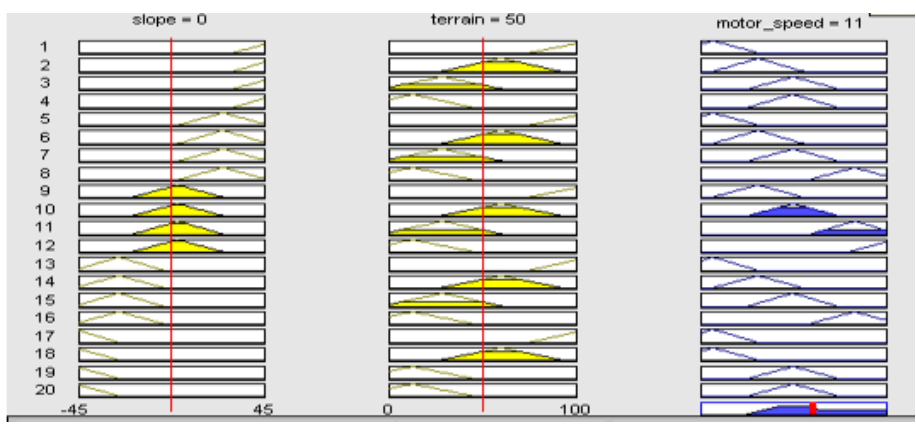

Fig.5 Rule view of Triangular shape membership function with five linguistic variables

\subsection{Trapezoidal shape membership function with 5 variables}

The shape of membership function considered in this case is trapezoidal. The membership function has five linguistic variables. Fig.6 shows the trapezoidal shape input membership function slope with five linguistic variables. Fig.7 shows Trapezoidal shape output membership function slope with five linguistic variables. Fig. 8 Rule view of Trapezoidal shape with five membership function variables. When the surface roughness value is 50 ,motor speed is 10.9 miles per hour

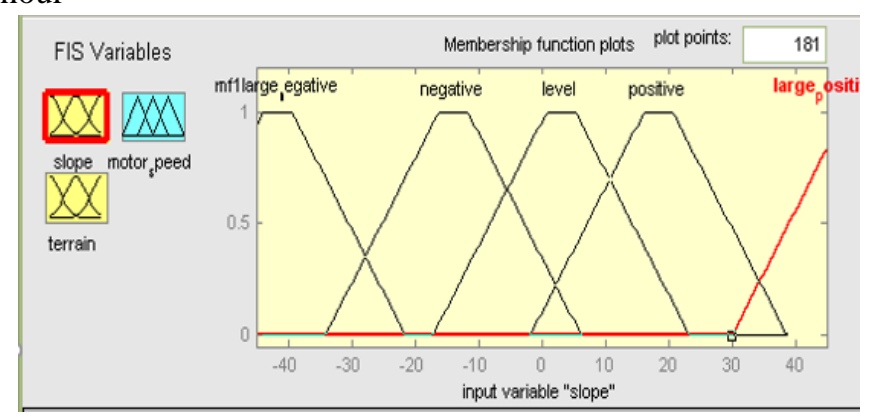

Fig.6 Trapezoidal shape input membership function slope with five linguistic variables

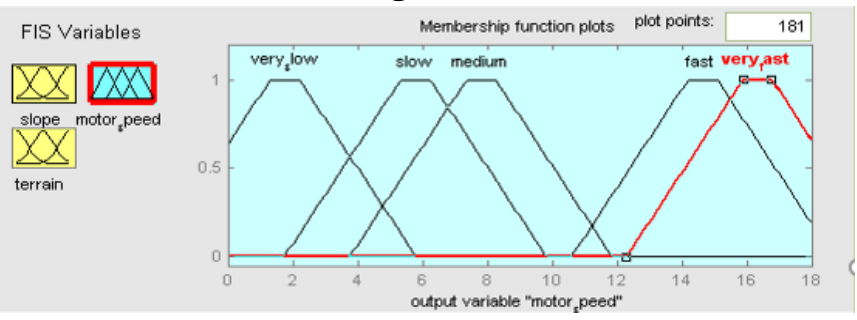

Fig.7 Trapezoidal shape output membership function slope with five linguistic variables

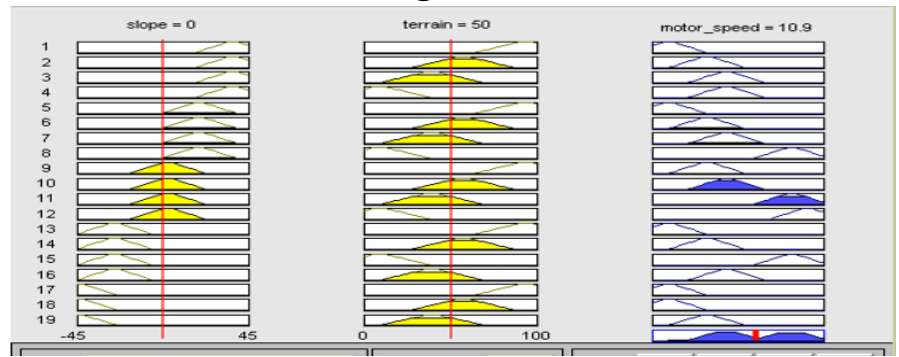

Fig.8 Rule view of Trapezoidal shape with five membership function variables 


\subsection{Gbell shape membership function with 5 variables}

The membership function considered in this case Gbell shape with five linguistic variables. Fig.8 Input slope in Gbell shape with five membership function variables. Fig.9 Output membership function motor speed in Trapezoidal shape with five membership function variables.Fig.10 shows rule view of Gbell shape membership function with five linguistic variables. When the surface roughness value is 50 ,motor speed is 8.89 miles per hour

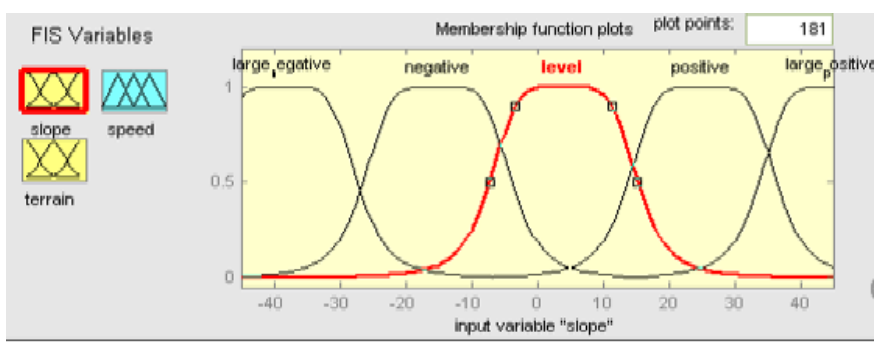

Fig.8 Input slope in Gbell shape with five membership function variables

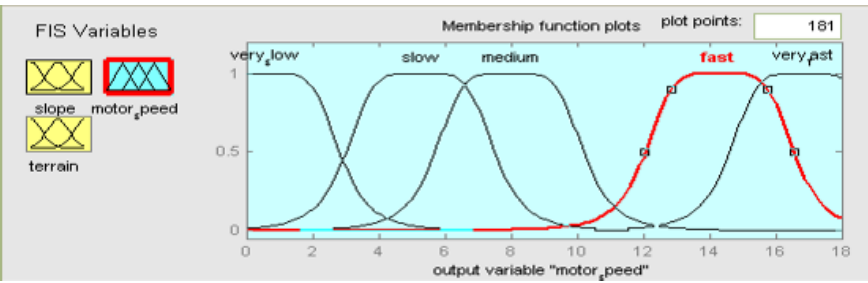

Fig.9 Output membership function motor speed in Gbell shape with five membership function variables

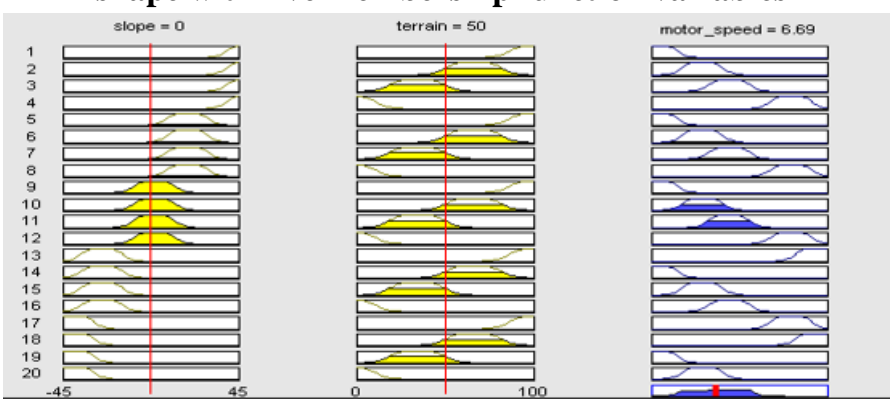

Fig.10 Rule view of Trapezoidal shape with five membership function variables

\subsection{Triangular shape membership function with 3 variables}

The membership function considered in this case Triangular shape with three linguistic variables. Fig.11 Input slope in triangular shape with 3 membership variables. Fig.9 Output membership function motor speed in Trapezoidal shape with three membership function variables.Fig.12 shows rule view of Gbell shape membership function with three linguistic variables. When the surface roughness value is 50 , motor speed is 8.89 miles per hour. Fig.13 Output motor speed in triangular shape with 3 membership function variables.

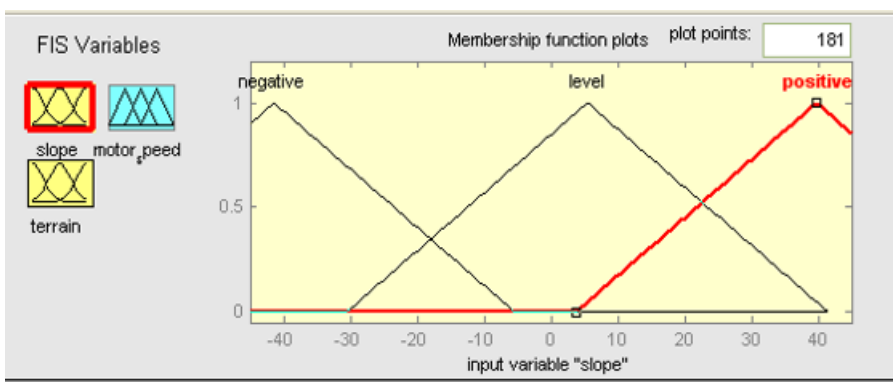

Fig.11 Input slope in triangular shape with 3 membership function variables

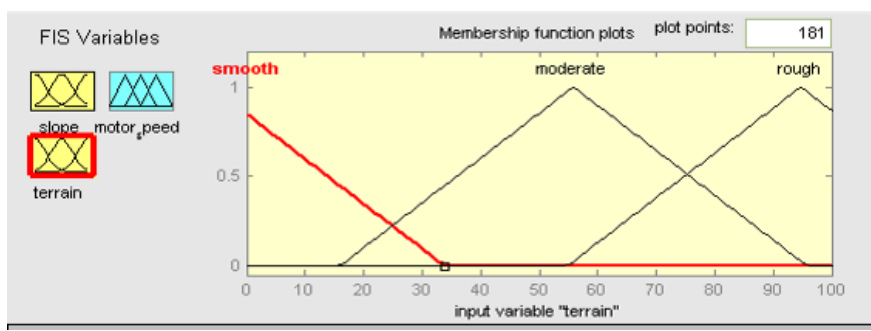

Fig.12 Input membership in triangular shape with 3 membership function variables

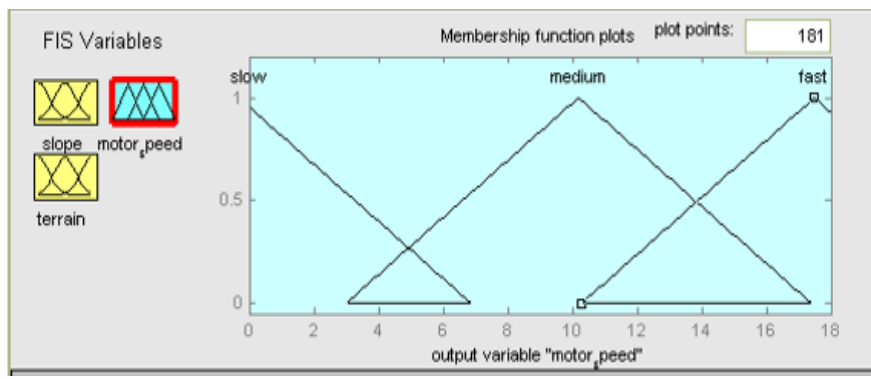

Fig.13 Output motor speed in triangular shape with 3 membership function variables

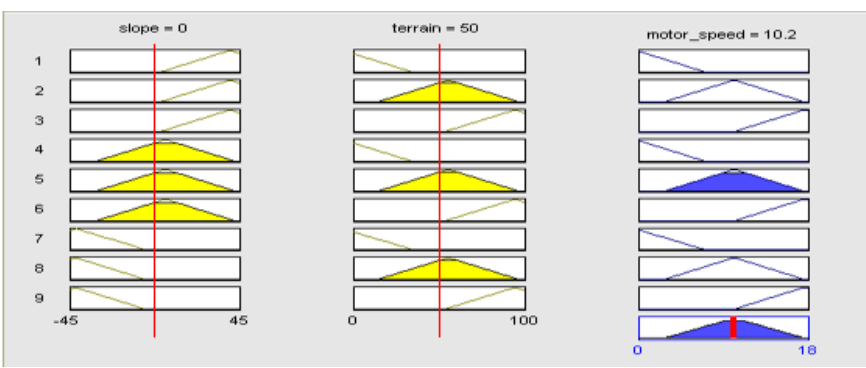

Fig.14 Rule view of Trapezoidal shape with three membership function variables

4.4 Triangular shape membership function with 3 linguistic variables

The membership function considered in this case Triangular shape with three linguistic variables. Fig.15 Input slope in triangular shape with 3 membership variables. Fig.16 Output membership function motor speed in Trapezoidal shape with three membership function variables.Fig. 17 shows rule view of triangular shape membership function with three linguistic variables. When the surface roughness value is 50 , 
motor speed is 9.67 miles per hour. Fig. 18 shows the rule view of triangular shape with three membership function variables

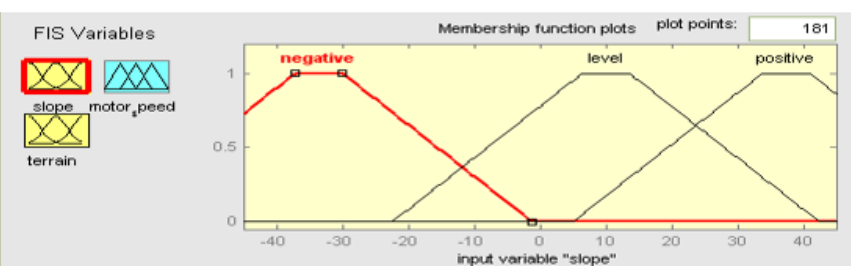

Fig.15 Input slope in trapezoidal shape with 3 membership function variables

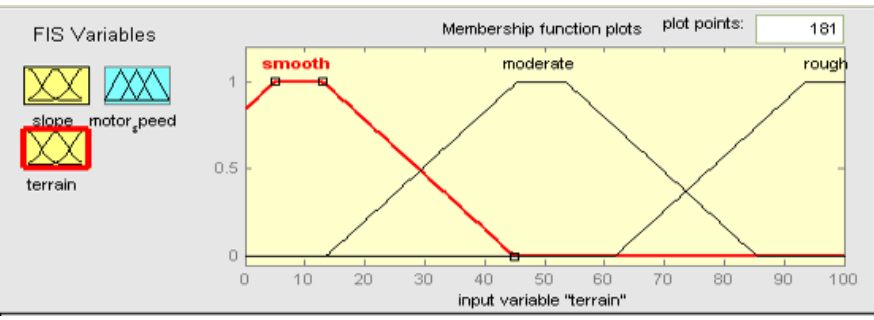

Fig.16 Input terrain type in trapezoidal shape with 3 membership function variables

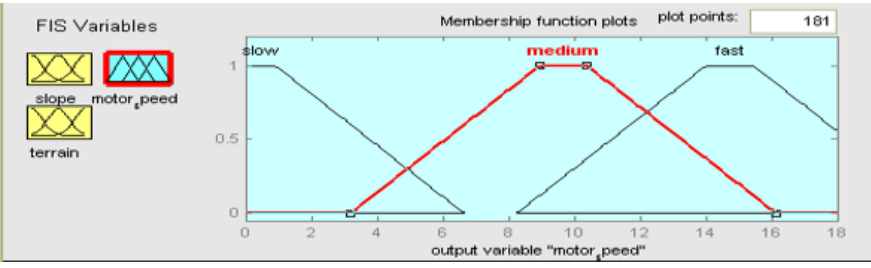

Fig.17 Output motor speed in trapezoidal shape with 3 membership function variables
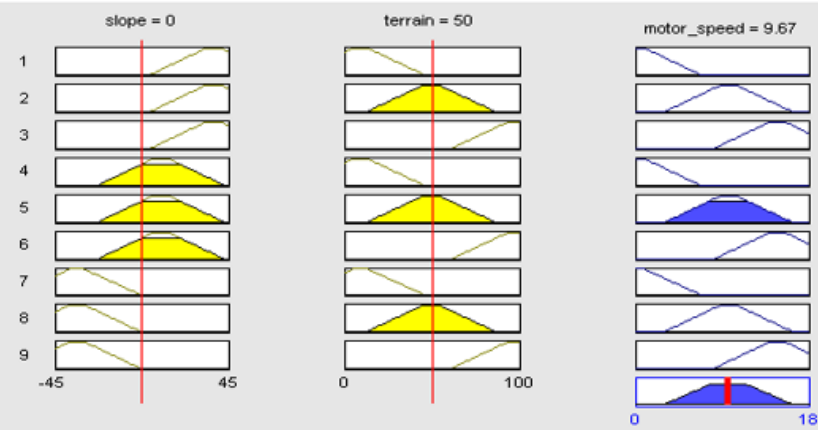

Fig.18 Rule view of Triangular shape with five membership function variables

4.5 Gbell shape membership function with 3 linguistic variables

The membership function considered in this case Gbell shape with three linguistic variables. Fig.19 Input slope in Gbell shape with 3 membership variables. Fig.20 Output membership function motor speed in Trapezoidal shape with three membership function variables.Fig. 21 shows rule view of Gbell shape membership function with three linguistic variables. When the surface roughness value is 50 ,motor speed is 9.67 miles per hour. Fig.22 shows the rule view of Triangular shape with five membership function variables.

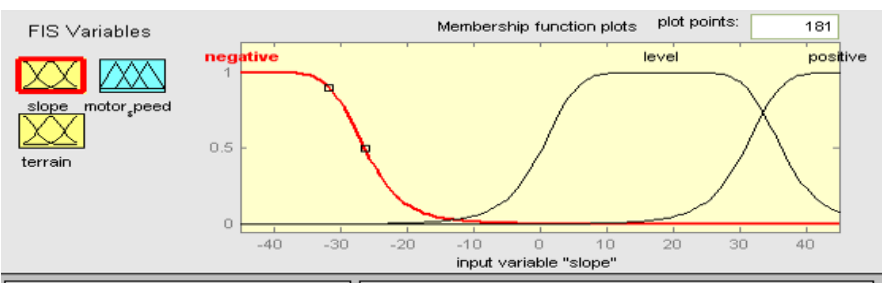

Fig.19 Input slope in Gbell shape with 3 membership function variables

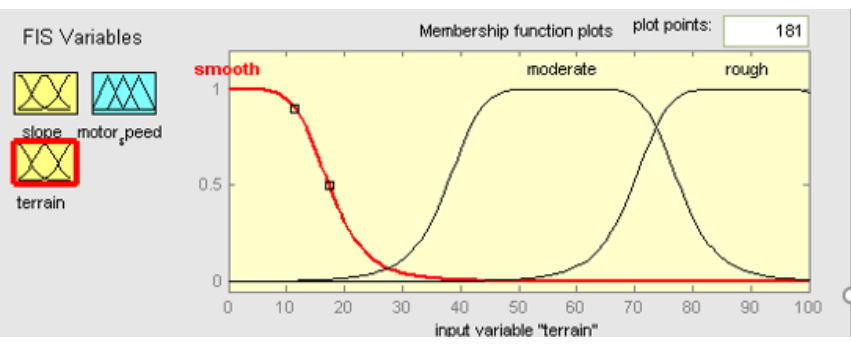

Fig.20 Input slope in Gbell shape with 3 membership function variables

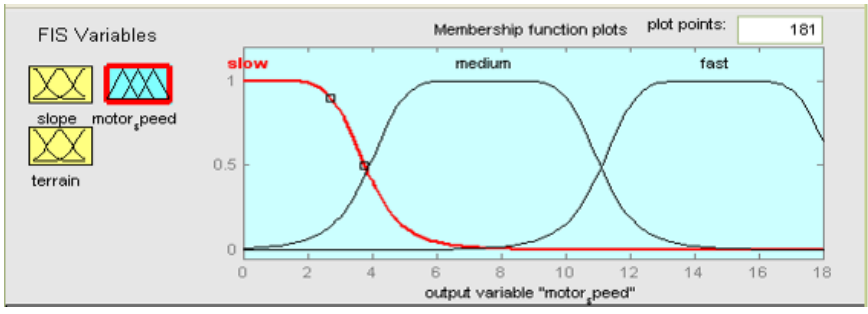

Fig.21 Input slope in Gbell shape with 3 membership function variables
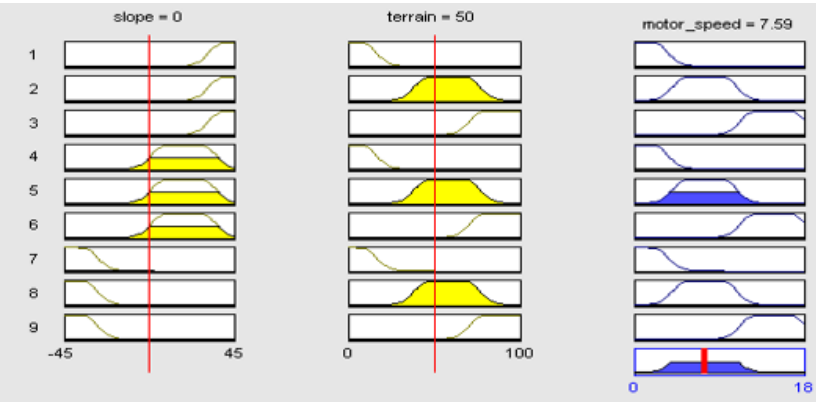

Fig.22 Rule view of Triangular shape with five membership function variables

\subsection{Gauss shape membership function with 3 linguistic} variables

The membership function considered in this case is Gauss shape with three linguistic variables. Fig.23 Input slope in Gauss shape with 3 membership variables. Fig.24 shows the output membership function motor speed in Gauss shape with three membership function variables.Fig. 25 shows rule view of Gauss shape membership function with three linguistic variables. When the surface roughness value is 50 ,motor speed is 9.67 miles per hour 


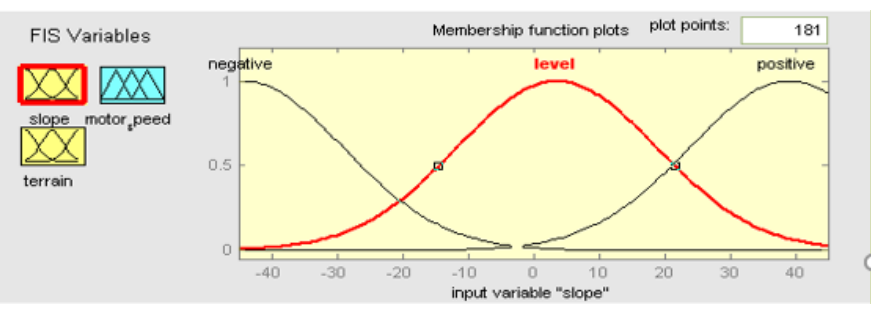

Fig.23 Input slope in Gauss shape with 3 membership function variables

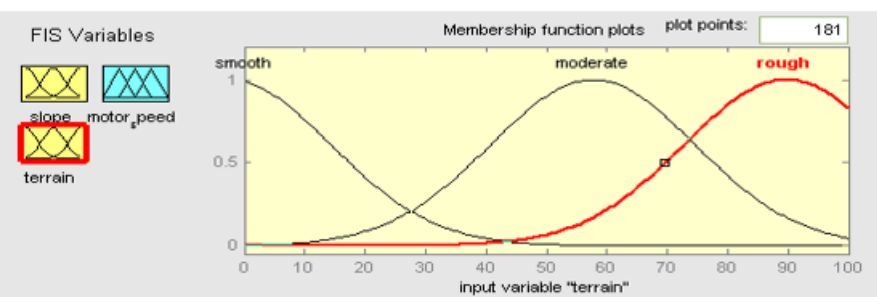

Fig.24 Input terrain type in Gauss shape with 3 membership function variables

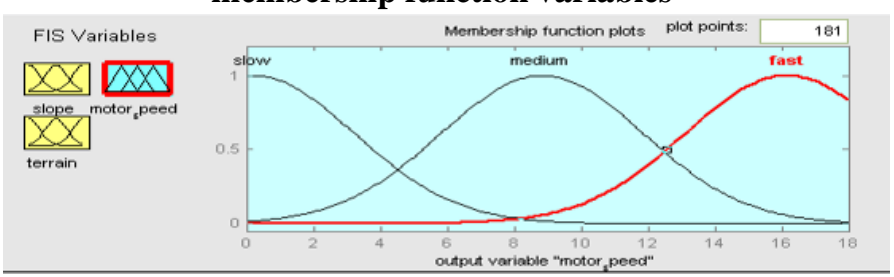

Fig.25 Input terrain type in Gauss shape with 3 membership function variables
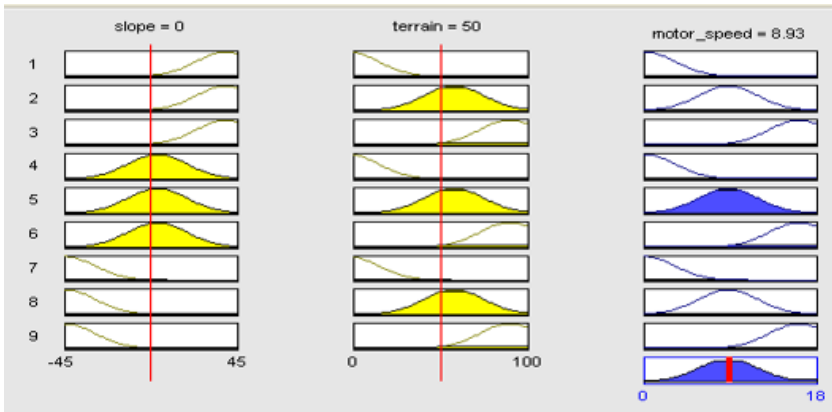

Fig.26 Rule view of Gauss shape with membership function variables

\section{Result and Discussion}

The different membership functions are used. It is evident that the fuzzy triangular membership function gives a maximum speed of 10.2 miles/hour and the Gbell membership function gives the least speed of 8.93 miles/hour.

\begin{tabular}{|l|c|c|c|}
\hline Membership & \multicolumn{2}{|c|}{ Input } & Output \\
\cline { 2 - 4 } Functions (MF) & $\begin{array}{c}\text { Slope of } \\
\text { terrain } \\
\text { (degree) }\end{array}$ & $\begin{array}{c}\text { Terrain } \\
\text { type } \\
\text { (ruggedness) }\end{array}$ & $\begin{array}{c}\text { Motor } \\
\text { speed } \\
\text { (miles/hour) }\end{array}$ \\
\hline Triangular & 0 & 50 & 10.2 \\
\hline Trapezoidal & 0 & 50 & 9.67 \\
\hline Gbell & 0 & 50 & 7.59 \\
\hline Gauss 1 & 0 & 50 & 8.93 \\
\hline
\end{tabular}

Table 1: Comparison of different membership functions

\section{Conclusion}

Fuzzy logic approach has been proved to be a simple and powerful technique for control problems. We here described the design process of fuzzy logic-based motion control on a rough terrain for a autonomous mobile robot. The robot motor speed was independently controlled in two case. In this work, the different kinds of membership functions of fuzzy logic controller simulation results presented by using the MATLAB tool. In first cases five membership function variables and twenty rules will be used and get the output range is close related to triangular MF, trapezoidal MF. In second cases only three membership function variables and nine rules will be followed but not close related to each other membership function. It is shown that fuzzy logic controller with five provides better performance compared with three membership function. In two cases, the slope of the terrain increases, terrain condition is very rough and the motor speed also increases in all membership function. The necessity of the simulation is very important for the development of the application and MATLAB is a useful and friendly tool for do that work. In future, a hybrid technique involving fuzzy and neural networks system could be explored to enhance the problems.

\section{References}

[1] Sawsan Abdel, Zaky B. Nossair, Hala M. Abdel-Kader Mansour, Fuzzy logic control of an Autonomous Mobile Robot ${ }^{\text {eee }}$ International conference on Methods \& Models in Automation and Robotics, vol. 2, pp.188 193, 2011.

[2] Ritesh B. Meshram, Motion Control of Wheeled Mobile Robot Using Fuzzy Logiceee International Journal of Recent Technology and engineering, vol.2, july 2013.

[3] Olumide OBE, Ioan Dumitrache, Fuzzy Control of Autonomous Mobile Robot ${ }^{\text {eee }}$ U.P.B.Sci, vol. $72,2010$.

[4] Leslie Astudilo, Oscar Castillo, Patricia Merlin, Intelligent Control of an Autonomous Robot Using Type-2 Fuzzy Logic ${ }^{\text {eee }}$ Advances in Soft Computing, vol. 41, pp.799 -807, August 2006.

[5] Xi Li, Byung-Jae Choi, Design of Obstacle Avoidance System for Mobile Robot using Fuzzy Logic Systems "ee International Journal of Smart Home, vol. 7, May 2013.

[6] Ricardo Alvaez Gonzalez, Definition and Simulation of Fuzzy Controllers for the motion of a Mobile Robot and the Force Exerted at the Robot $^{\text {ee }}$ s Hand ${ }^{\text {eec }}$ International Conferences. 
[7] Umar Farooq, Muhammad Amar, Athar Hanif, Fuzzy Logic Reasoning System for Line Following Robot "ee International Journal of Engineering and Technology, vol.6, August 2014.

[8] Mohammed Faisal, Ramdane Hedjar, Mansour Al Sulaiman, Fuzzy Logic Navigation and Obstacle Avoidance by a Mobile Robot in an Unknown Dynamic Environment eee International Journal of Advanced Robotic Systems, April 2012

[9] Nitin Afzulpurkar, Rerngwut Choomuang, Fuzzy Logic based Position Control of Autonomous Mobile Robot eee International Journal of Advanced Robotic Systems, vol. 2, pp.197 - 208, 2005.

[10] D.Elayaraja, Ramabalan, Fuzzy Logic based Motion Control of Mobile Robot in a Rough Terrain 'International Conference on Advances in Engineering,Science and Management (ICAESM), March 2012.

[11] Harisha S.K, Ramakanth P. Kumar, M. Krishna, Development of Hardware Simulator to Validate Free-State based Navigation Algorithm of AGV'" International Journal of Current Engineering and Technology, vol. 3, August 2013

[12] Mohammed Faisal, Khalid Al-Mutib, Hasan Mathkour, Multi Modules Fuzzy Logic for Mobile Robots Navigation and Obstacle Avoidance in Unknown indoor Dynamic Environment 'International Conference on Systems, September 2013. 